NASA/TM-2005-213816

\title{
Near-Field Noise Computation for a Supersonic Circular Jet
}

Ching Y. Loh

Taitech, Inc., Beavercreek, Ohio

Lennart S. Hultgren

Glenn Research Center, Cleveland, Ohio 
Since its founding, NASA has been dedicated to the advancement of aeronautics and space science. The NASA Scientific and Technical Information (STI) Program Office plays a key part in helping NASA maintain this important role.

The NASA STI Program Office is operated by Langley Research Center, the Lead Center for NASA's scientific and technical information. The NASA STI Program Office provides access to the NASA STI Database, the largest collection of aeronautical and space science STI in the world. The Program Office is also NASA's institutional mechanism for disseminating the results of its research and development activities. These results are published by NASA in the NASA STI Report Series, which includes the following report types:

- $\quad$ TECHNICAL PUBLICATION. Reports of completed research or a major significant phase of research that present the results of NASA programs and include extensive data or theoretical analysis. Includes compilations of significant scientific and technical data and information deemed to be of continuing reference value. NASA's counterpart of peerreviewed formal professional papers but has less stringent limitations on manuscript length and extent of graphic presentations.

- TECHNICAL MEMORANDUM. Scientific and technical findings that are preliminary or of specialized interest, e.g., quick release reports, working papers, and bibliographies that contain minimal annotation. Does not contain extensive analysis.

- CONTRACTOR REPORT. Scientific and technical findings by NASA-sponsored contractors and grantees.
- CONFERENCE PUBLICATION. Collected papers from scientific and technical conferences, symposia, seminars, or other meetings sponsored or cosponsored by NASA.

- SPECIAL PUBLICATION. Scientific, technical, or historical information from NASA programs, projects, and missions, often concerned with subjects having substantial public interest.

- TECHNICAL TRANSLATION. Englishlanguage translations of foreign scientific and technical material pertinent to NASA's mission.

Specialized services that complement the STI Program Office's diverse offerings include creating custom thesauri, building customized databases, organizing and publishing research results ... even providing videos.

For more information about the NASA STI Program Office, see the following:

- Access the NASA STI Program Home Page at http://www.sti.nasa.gov

- E-mail your question via the Internet to help@sti.nasa.gov

- Fax your question to the NASA Access Help Desk at 301-621-0134

- Telephone the NASA Access Help Desk at 301-621-0390

- Write to:

NASA Access Help Desk

NASA Center for AeroSpace Information 7121 Standard Drive

Hanover, MD 21076 
NASA/TM-2005-213816

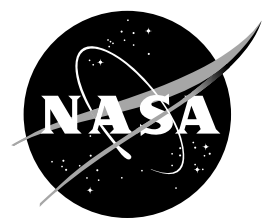

\section{Near-Field Noise Computation for a Supersonic Circular Jet}

Ching Y. Loh

Taitech, Inc., Beavercreek, Ohio

Lennart S. Hultgren

Glenn Research Center, Cleveland, Ohio

Prepared for the

11th Aeroacoustics Conference

cosponsored by the American Institute of Aeronautics and Astronautics and the Confederation of European Aerospace Societies

Monterey, California, May 23-25, 2005

National Aeronautics and

Space Administration

Glenn Research Center 


\section{Acknowledgments}

The authors thank Jayanta Panda for supplying his experimental data and insightful discussions. This work received support from the Quiet Aircraft Technology Project Office at NASA Glenn Research Center.

This report contains preliminary

findings, subject to revision as analysis proceeds.

Available from

NASA Center for Aerospace Information 7121 Standard Drive

Hanover, MD 21076
National Technical Information Service 5285 Port Royal Road Springfield, VA 22100 


\title{
NEAR-FIELD NOISE COMPUTATION FOR A SUPERSONIC CIRCULAR JET
}

\author{
Ching Y. Loh and Lennart S. Hultgren \\ National Aeronautics and Space Administration \\ Glenn Research Center \\ Cleveland, Ohio 44135
}

\begin{abstract}
A fully expanded, high-Reynolds-number, supersonic circular jet of Mach number 1.4 is simulated, using a 3-D finite-volume NavierStokes solver, with emphasis on the near field noise. The numerical results are generally in good agreement with existing experimental findings.
\end{abstract}

\section{Introduction}

The reduction of aircraft noise is an important technological challenge as a result of the environmental limitations and economical consequences of airport noise pollution. Jet noise, a major noise component for modern commercial aircraft engines, consists of turbulent mixing noise and, in the case of imperfectly expanded supersonic jets, broadband shock-associated noise (as well as, discrete screech tones under certain circumstances). Jet noise has been a vital part of aeroacoustics since the early 1950s and continues to be a topic of many experimental and theoretical investigations; Refs. [1-3] provide a comprehensive discussion and further references. A rapid advance in its understanding has occurred during the last decade, perhaps in conjunction with the development of computational aeroacoustics (CAA), as well as better measurement techniques. The issues, challenges, and contributions of CAA are discussed in Refs. [4-6], and the Rayleigh-scattering technique [7-9] is an example of advances in experimental methods for high-speed jets.

For supersonic jet mixing noise, it is generally agreed that there are two different sources [10-12]. One is associated with the convection/evolution of large-scale turbulence structures in the jet and which produces intense sound propagating downstream at angles close to the jet axis. Traditionally, this has been referred to as eddy Mach wave radiation. The other noise source is associated with the fine scale turbulence in the jet shear flow and which produces sound propagating predominantly in the lateral $\left(90^{\circ}\right)$ direction [13-16]. The work in this paper concerns the sound produced by the large-scale structures in the jet.

Substantial progress in numerical computation of jet mixing noise associated with the large-scale structures has been made during recent years by using large-eddy simulation (LES) techniques. Mankbadi et al. [17] performed an axisymmetric LES computation of a supersonic, high-Reynolds-number jet and determined its farfield radiated sound using an acoustic analogy technique. Zhao et al. [18], Bogey et al. [19], Uzun et al. [20] computed jet flow and the associated noise by using fully 3D LES at a Mach number of 0.9. The Reynolds number in these simulations were low to moderate, however, $5,000,65,000$, and 100, 000, respectively. DeBonis and Scott [21] used LES to calculate the hydrodynamics of a high-Reynolds-number, supersonic jet of Mach number $1.4[8,9]$. In all of the above work, the numerical schemes are of high-order finite-difference type.

In the present paper, encouraged by the successful computation of 2-D axisymmetric and 3-D screech noise for round jets [22-25], a second-order (in space and time) finite-volume scheme of low dissipation is adopted for LES computation of a supersonic jet of Mach number 1.4. The scheme is the 3-D CE/SE Navier-Stokes solver using a hexahedral grid.

\section{The 3-D Supersonic Jet Noise Problem}

The current computation is for the conditions in the Panda and Seasholtz $[8,9]$ experiments on a circular, high-Reynolds-number, supersonic, cold jet at Mach number 1.4. In these experiments, a nominally fully expanded jet is issuing from a convergent-divergent (C-D) nozzle.

In what follows, the exit diameter $D$ of the of the nozzle, the density $\rho_{\infty}$, the speed of sound $a_{\infty}$, and the temperature $T_{\infty}$ in the ambient fluid are used as scales to make all the dependent variables nondimensional. Us- 
ing the ideal gas isentropic relations and conservation of mass gives that the reservoir and jet exit Mach numbers are given by

$$
\begin{gathered}
M_{r}=\left(A_{e} / A_{r}\right)\left(P_{e} / P_{r}\right)^{(\gamma+1) / 2 \gamma} M_{e}, \\
M_{e}=\sqrt{\frac{2\left[\left(P_{r} / P_{e}\right)^{(\gamma-1) / \gamma}-1\right]}{(\gamma-1)\left[1-\left(A_{e} / A_{r}\right)^{2}\left(P_{e} / P_{r}\right)^{2 / \gamma}\right]}},
\end{gathered}
$$

respectively, where the subscripts 'e' and ' $r$ ' denote jetexit and reservoir conditions, $P$ is the nondimensional static pressure, $A_{r} / A_{e}$ is the area ratio of the nozzle, and $\gamma$ is the constant specific heat ratio. Since the jet is nominally fully expanded

$$
P_{e}=1 / \gamma .
$$

Furthermore, it also follows that

$$
\begin{gathered}
\rho_{e}=1 / T_{e}, \\
T_{e}=T_{r}\left(1+\frac{\gamma-1}{2} M_{r}^{2}\right) /\left(1+\frac{\gamma-1}{2} M_{e}^{2}\right), \\
u_{e}=M_{e} \sqrt{T_{e}}, \\
\rho_{r}=\gamma P_{r} / T_{r}, \\
u_{r}=M_{r} \sqrt{T_{r}},
\end{gathered}
$$

where $\rho, T$, and $u$ denote the nondimensional density, static temperature and axial velocity, respectively. In the experiment [8], $P_{r} / P_{e}=3.16, T_{r}=1.01$, and $A_{r} / A_{e}=9$, which together with (1)-(8) largely define the experimental conditions.

\section{Numerical Method}

In this work, the three-dimensional numerical scheme is designed to solve an LES version of the compressible Navier-Stokes equations. The numerical scheme adopted in this work is a variation of the low-dissipation finitevolume $\mathrm{CE} / \mathrm{SE}$ scheme. The CE/SE scheme has formal second-order accuracy in both space and time [26, 27] and naturally captures shocks and other discontinuities in the flow.

\subsection{Computational Domain and Unstructured Hexahedral Grid}

The computational domain including the C-D nozzle is sketched in Fig. 1. The numerical simulation begins at the inlet of the C-D nozzle. The geometrical specification of the C-D nozzle is given in $[8,9]$. In nondimensional form, the C-D nozzle extends from $x=-4.9$ to $x=0$ and the external domain ranges from $x=-0.2$ to $x=17$, where $\mathrm{x}$ is the streamwise coordinate. The external domain diameter is about 10.8 .

For fully 3-D computations of complicated problems such as the current jet noise problem where several distinct and disparate length scales need to be resolved [4],

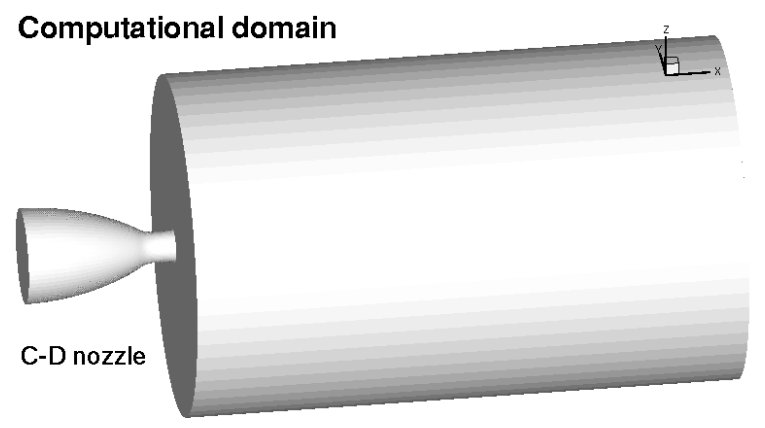

Figure 1: Geometry of the computational domain, comprising the interior of the C-D nozzle and an external domain; the nozzle extends into the external domain by two internal nozzle exit diameters.

a large number of computational cells are needed to provide sufficient numerical resolution. The choice of an unstructured hexahedral grid for the CE/SE method leads to a significant reduction of the number of computational cells, with an associated reduction in computer memory and CPU time requirements, as compared to the commonly used tetrahedral one. The drawback is a modest increase in numerical dissipation.

The unstructured hexahedral grid is generated in the following manner [30]: first, a 2-D unstructured quadrilateral grid is generated in a circular region (Fig. 2) and then the circular disc is advanced one step in the streamwise direction with step size $\Delta x$. The advancing front technique is carried until $x=17$. The step size $\Delta x$ ranges from 0.02 at the nozzle exit $(x=0)$ to about 0.06 at $x=15$. Between $x=15$ and $x=17$ there is a buffer zone in which $\Delta x$ increases exponentially in size to provide high numerical damping. Inside the $\mathrm{C}$ D nozzle, $\Delta x$ is much refined. Totally, there are 3.17 million cells in the computational domain, including the the buffer zone. The domain covers the near-field region which is the source region for the noise.

Employing a hexahedral grid instead of a tetrahedral one for the current $\mathrm{CE} / \mathrm{SE}$ scheme helps to reduce the number of cells from tens of millions to a few millions. Still, the number of computational cells is very large and the simulation is implemented using parallelcomputation techniques. To accomplish this, the unstructured hexahedral grid generated for the entire computational domain is decomposed into subdomains (12 at present) using the efficient mesh partitioning code METIS [28]. Figure 3 illustrates a typical partition of the computational domain for the current circular jet noise problem. The CE/SE flow solver uses message-passinginterface (MPI) library calls to exchanging pertinent information between neighboring subdomains during the simulation. 


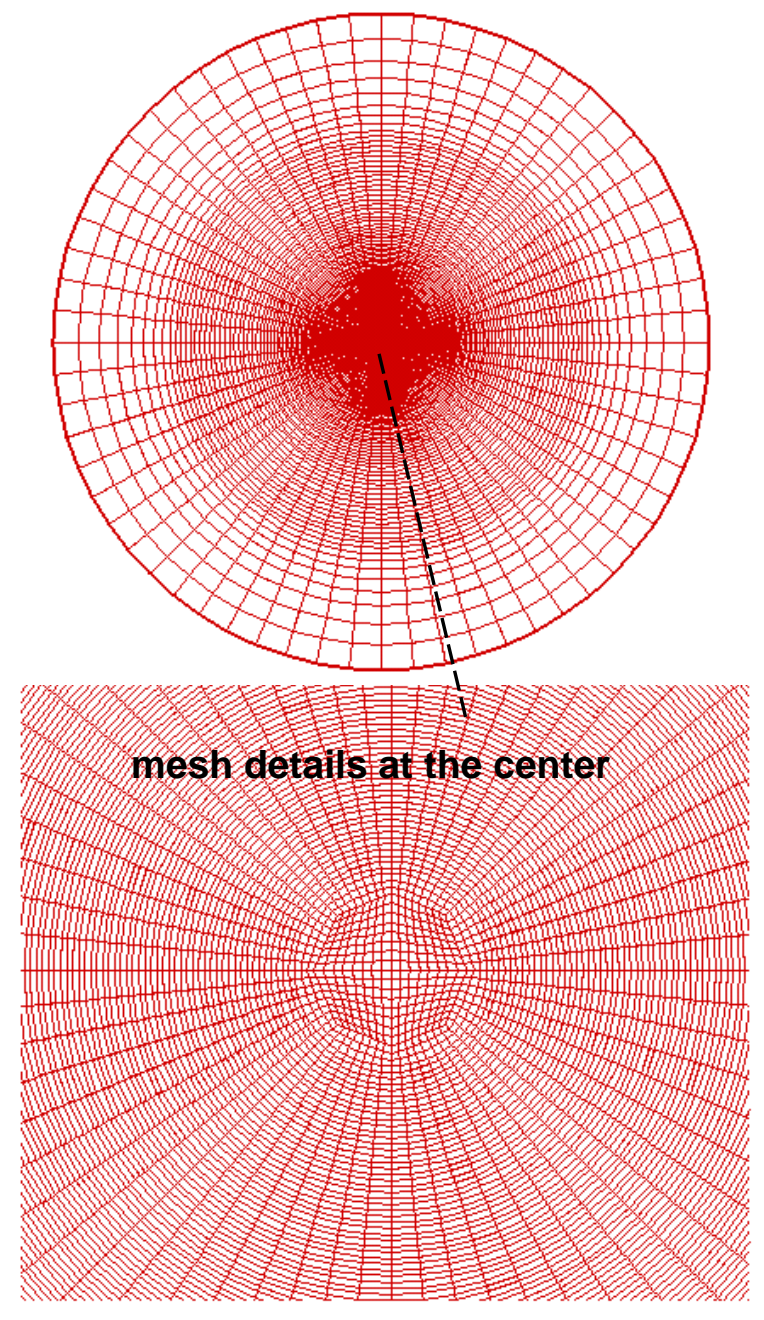

Figure 2: 2-D quadrilateral mesh in a cross section in a 3-D hexahedral grid

\subsection{Conservation Form of the 3-D Unsteady Compressible Navier-Stokes Equations}

The Navier-Stokes equations for a perfect gas can be written in the following nondimensional vector form, e.g. [29]:

$$
\boldsymbol{U}_{t}+\boldsymbol{F}_{x}+\boldsymbol{G}_{y}+\boldsymbol{H}_{z}=\mathbf{0}
$$

where $x, y$, and $z$ are the streamwise, transverse, and spanwise coordinates, and $t$ is time. The five components of the conservative flow variable vector $\boldsymbol{U}$ are: the density, the three momentum components, and the total energy per unit volume, i.e.,

$$
\begin{gathered}
U_{1}=\rho, \quad U_{2}=\rho u, \quad U_{3}=\rho v, \quad U_{4}=\rho w, \\
U_{5}=p /(\gamma-1)+\rho\left(u^{2}+v^{2}+w^{2}\right) / 2,
\end{gathered}
$$

where, as before, $\rho, u$, and $p$ denote the density, streamwise velocity, and static pressure, and $v$ and $w$ denote

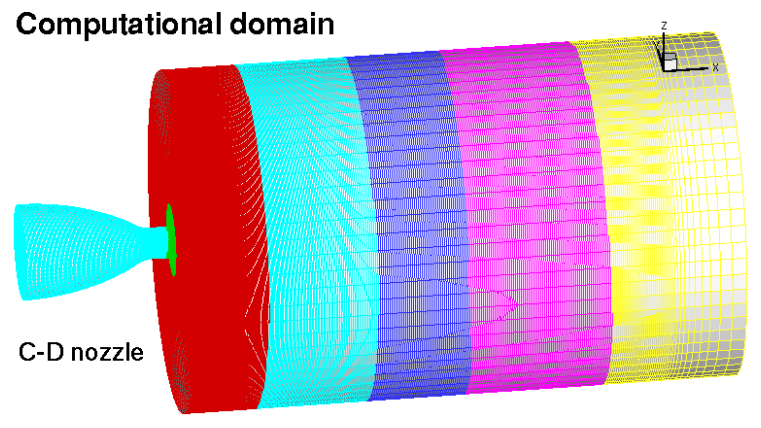

Figure 3: Partition of the computational domain for parallel computation

the transverse and spanwise velocities. The flux vectors in the $x, y$, and $z$ directions, $\boldsymbol{F}, \boldsymbol{G}$, and $\boldsymbol{H}$, respectively, have both inviscid and viscous contributions and the details of these flux components may be found in a textbook [29] or in a previous paper [30] by the present authors. Note that in the latter, $\mu$ is to be interpreted here as the nondimensional viscosity.

The nondimensional equations above could form the basis for a direct numerical simulation (DNS). Currently, this is not feasible for the high-Reynolds-number flows of interest here. The present computations are of the LES type, where the effects of the unresolved scales, i.e., scales smaller than the grid resolution, are modeled. In this case, the equations above are considered the filtered ones governing the resolved scales, where $\rho$ and $p$ are interpreted as simply averaged and the other dependent variables are interpreted as Favré, (i.e., densityweighted) averaged over the subgrid scale. The simplest model for the effects of the unresolved scales, or subgrid scales (SGS), on the motion is obtained by using a Boussinesq eddy-diffusivity assumption for the subgrid shear stresses and heat flux coupled by Smargorinsky's model for the eddy viscosity (eg. [31]). That is, $\mu$ occuring in the viscous contribution to the flux terms in Eq. (9) is replaced by

$$
\mu=1 / R e+\left(C_{s} \delta\right)^{2}\left(S_{i j} S_{i j}\right)^{1 / 2},
$$

where $S_{i j}=\frac{1}{2}\left(\frac{\partial u_{i}}{\partial x_{j}}+\frac{\partial u_{j}}{\partial x_{i}}\right)$ is the Favré averaged rateof-strain tensor, $\delta=(\delta x \delta y \delta z)^{1 / 3}$ is a local measure of the grid size, $C_{s}$ is a (nondimensional) constant, and $R e=a_{\infty} D / \nu_{\infty}$ is the Reynolds number for the computation. The inverse Reynolds number $1 / R e=1.8 \times 10^{-6}$ in the present computations, which corresponds to the value for the jet Reynolds number, $R e_{j}=u_{e} D / \nu_{e}$, of $1.2 \times 10^{6}$ in the experiments [8,9]. Also, note that the simplifying assumption is also made that the subgridscale Prandtl number equal the laminar one, which is taken to have the value 0.72 . 
By considering $(x, y, z, t)$ as coordinates of a fourdimensional Euclidean space, $E_{4}$, and using Gauss' divergence theorem, it follows that Eq. (9) is equivalent to the following integral conservation law:

$$
\oint_{S(V)} \boldsymbol{I}_{m} \cdot \mathrm{d} \boldsymbol{S}=\mathbf{0}, \quad m=1,2, \ldots, 5,
$$

where $S(V)$ denotes the surface around a volume $V$ in $E_{4}$ and $\boldsymbol{I}_{m}=\left(F_{m}, G_{m}, H_{m}, U_{m}\right)$. These equations are discretized and solved using a modified verison of the weighted-average $\mathrm{CE} / \mathrm{SE}$ scheme with a weghting factor $\alpha=0.8$.

Note that Fureby and Grinstein [32] point out that applying flux limiters to finite-volume methods (as is done in the current $\mathrm{CE} / \mathrm{SE}$ scheme), even in the absence of any explicit LES assumptions, effectively leads to LES schemes with minimal implicit SGS models. They demonstrated through an 'error' analysis of a particular scheme that the flux-limiters (essentially low-pass frequency filters) build into the algorithm produces additional terms in the equivalent differential forms of the momentum and energy equations that can be interpreted as the SGS stress tensor and flux, respectively. Hence, there is an additional implicit SGS model inherent in the particular CE/SE scheme used in addition to the explicit assumption in Eq. (10).

\subsection{Initial and Boundary Conditions}

Initially, the flow of the entire computational domain is set at the ambient flow conditions, i.e.,

$$
\begin{gathered}
\rho_{0}=1, \quad p_{0}=\frac{1}{\gamma}, \\
u_{0}=0, \quad v_{0}=0, \quad w_{0}=0 .
\end{gathered}
$$

At the exterior upstream inlet boundary, the conservative flow variables are specified to be the same as the ambient flow and their spatial derivatives are held at zero. At the nozzle inlet, the reservoir conditions as described in $\S 2$ are imposed. Of course, $v_{r}, w_{r}$, and all spatial derivatives are held at zero.

Type I CE/SE non-reflecting boundary conditions $[33,34]$ are imposed at the circumferential and outflow boundaries. The no-slip boundary condition is applied on all the nozzle walls, external as well as internal.

\subsection{Artificial Forcing}

As is common, e.g. [19], in numerical studies of jet mixing noise, the simulation allows for a low-level, artificial excitation, or turbulence seeding, of the jet shear layer near the nozzle exit. This seeding is accomplished by adding divergence-free velocity perturbations inside the jet shear layer at $x=0.3$. The disturbances are temporally random and are of the vortex-ring form used in [19], namely

$$
\begin{gathered}
u^{\prime}=\frac{r-r_{p}}{r} \mathcal{F}\left(x, r, \theta ; x_{p}, r_{p}\right), \\
\left(\begin{array}{c}
v^{\prime} \\
w^{\prime}
\end{array}\right)=-\frac{x-x_{p}}{r} \mathcal{F}\left(x, r, \theta ; x_{p}, r_{p}\right)\left(\begin{array}{c}
\cos \theta \\
\sin \theta
\end{array}\right),
\end{gathered}
$$

where

$$
\begin{gathered}
\mathcal{F}\left(x, r, \theta ; x_{p}, r_{p}\right)= \\
\frac{2 \delta_{p} r_{p}}{\Delta_{p}} \exp \left(-d^{2} \ln 2 / \Delta_{p}^{2}\right) \sum_{n=1}^{16} \epsilon_{n} \cos \left(n \theta+\phi_{n}\right)
\end{gathered}
$$

and $r=\left(y^{2}+z^{2}\right)^{1 / 2}$ and $\theta=\sin ^{-1}(z / r)$ are the radial and azimuthal coordiates; $x_{p}=0.3, r_{p}=0.5$ and $\Delta_{p}=0.02$ define the location and spatial extent of the perturbation, and $d=\left[\left(x-x_{p}\right)^{2}+\left(r-r_{p}\right)^{2}\right]^{1 / 2} ; \epsilon_{n}$ and $\phi_{n}$ are uniformly distributed random numbers between -1 and 1 , and 0 and $2 \pi$, respectively. They represent the random perturbation amplitude and phase, and are generated at every time step. The overall perturbation amplitude $\delta_{p}$ is either set to zero (no forcing) or to 0.01 in the present computations. The perturbations above are then directly added to the velocity field in the latter case.

\section{Numerical Results}

The simulation starts from the uniform initial conditions described in $\S 3.3$, without any artificial forcing. The simulation is first run 100,000 time steps, or to $t=100$ with the time step $\Delta t=0.001$ used, to ensure that all start-up transients are convected out of the computational domain and that the jet flow is fully established. This time, $t=100$, corresponds roughly to five acoustic times. The acoustic time is a measure of how long it takes a sound wave to pass through the external computational domain. The simulation is then run for an additional 340, 000 time steps to $t=440$, or approximately an additional 17 acoustic times, to achieve sufficient accuracy in the spectral analysis of time series data that is saved at various locations in the computation domain every 20 time steps. The artificial forcing is then turned on with an amplitude $\delta_{p}=0.01$ and the simulation is again run 100, 000 time steps to remove any transients. The simulation is then run an additional 350, 000 time steps, with the random turbulence seeding in the jet shear layer in the vicinity of the nozzle lip in effect, and time series data is saved at the same locations as in the unforced case, again every 20 time steps. The forced and unforced results are compared below.

\subsection{Time-Averaged Data}

Figure 4 shows a comparison of the evolution of the time-averaged centerline streamwise velocity between the simulation (solid and dashed lines) and the experiments $[8,9]$. In general, the agreement is good, particularly for the forced case, but with the end of the potential 


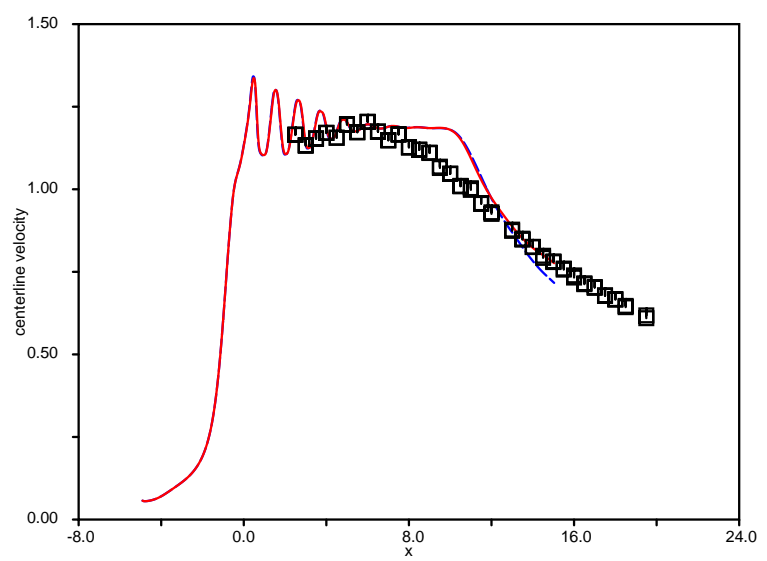

Figure 4: Time-averaged centerline streamwise velocity evolution; solid line: computation with artificial forcing, dashed line: computation with no forcing, symbols: experiment

core predicted by the simulation occurring somewhat too far downstream.

Figure 5 shows comparisons of time-averaged radial density profiles from the simulations (solid and dashed lines) with experimental results [8,9] for six streamwise stations, $x=2,4, \ldots, 12$. The agreement for the first two stations ( $x=2$ and 4 , respectively) is very good. This indicates that the early jet shear layers are quite well resolved in the simulations. The agreement for $x=6$ is still reasonable, but it is poor for $x=8$ and 10 , reflecting the discrepancy also seen in Fig. 4 at these stations. However, the agreement is again quite good for $x=12$. This suggests that the simulation captures the more energetic/violent mixing process that occurs after the potential core is gone.

\subsection{Instantaneous Flow Fields}

Figure 6 shows the computed instantaneous u-velocity field in the spanwise plane, $y=0$, at $t=440$ and $t=890$ for the no-forcing (top) and random-forcing (bottom) cases, respectively. The rather weak shock-cell like structures in the upstream part of the potential core indicate that the expansion is not $100 \%$ perfect as a result of either the C-D nozzle design, or the numerical discretization/implementation in the nozzle, or both. The potential core is observed to end around 10 diameters downstream of the nozzle exit and the mixing process then rapidly gains strength. Comparing the two plots in the figure, the instantaneous flows look qualitatively similar.

Figure 7 depicts the computed instantaneous spanwise vorticity, $\omega_{y}$, in the same spanwise plane, $y=0$, and times $t=440$ and $t=890$ as in the previous figure.
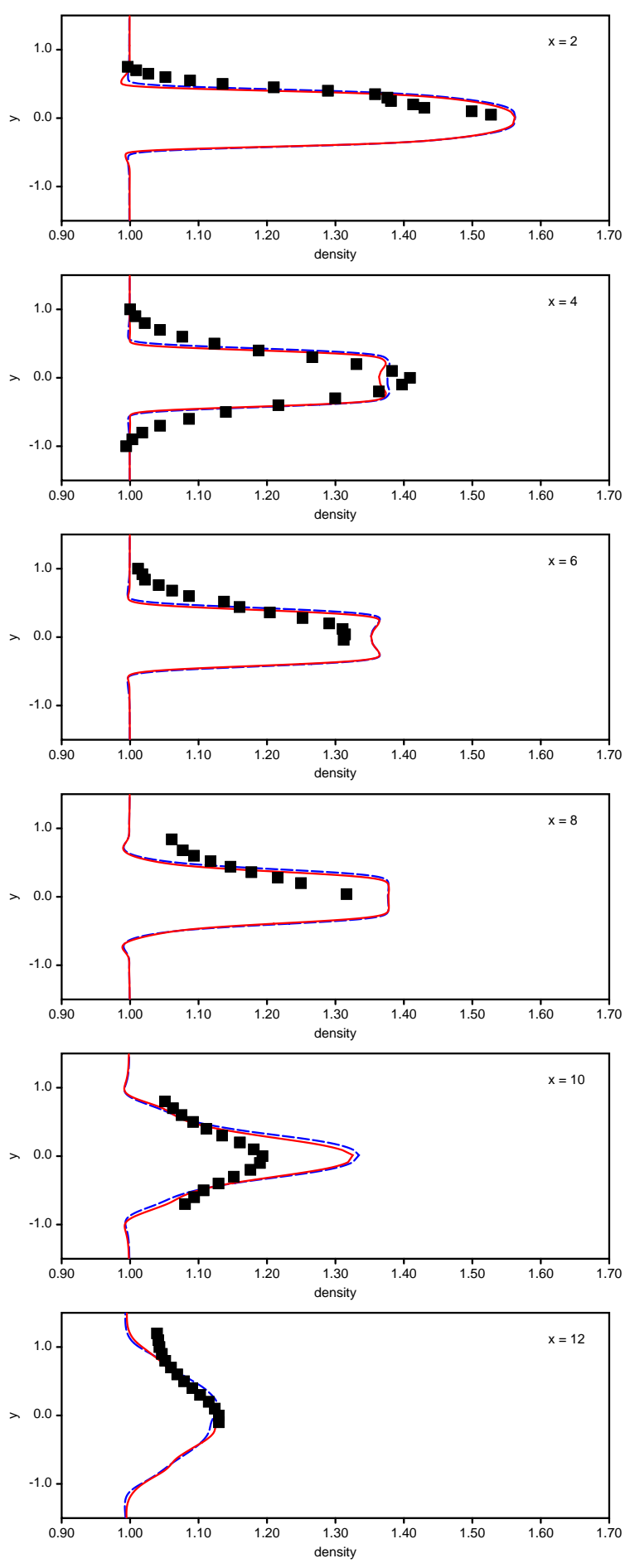

Figure 5: Radial variation of time-averaged density at six streamwise locations, $x=2,4, \ldots, 12$; solid line: computation with artificial forcing, dashed line: computation with no forcing, symbols: experiment 
The instantaneous flow in the case with random forcing (bottom) seems more mixed. Otherwise, the mixing processes qualitatively look quite similar in both plots.

Figure 8 shows the computed instantaneous streamwise vorticity $\omega_{x}$ in four streamwise (or cross-sectional) planes, $x=3.75,7.5,11.25$, and 15 . The top panel is the case without forcing at $t=440$ and the bottom panel is with random forcing at $t=890$. These $\omega_{x}$ plots clearly show, in both cases, the 3-D nature of the unsteady motion at all stations. Again, the unsteady motion is qualitatively similar in both cases.

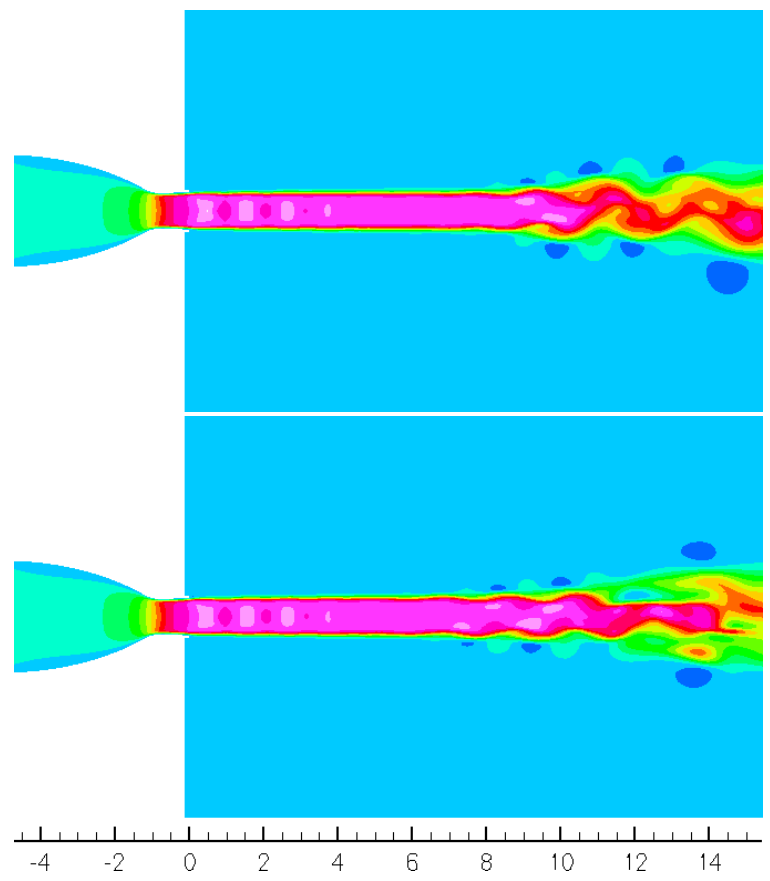

Figure 6: Instantaneous streamwise velocity distribution in a spanwise plane; top, no forcing at $t=440$; bottom, random forcing at $t=890$

Figure 9 shows the computed instantaneous 3-D pressure isosurfaces at $t=440$ (without forcing) and $t=$ 890 (random forcing) at the two levels of $p=0.71$ and $p=0.715$. Note that the nondimensional ambient pressure is $1 / 1.4$. The isosurfaces show downstream radiating 3-D sound waves caused by the large-scale coherent structures in the jet flow, i.e. eddy-Mach wave radiation. In the bottom plot, artifacts of the random forcing can be seen in the vicinity of the nozzle exit, but these are rapidly washed out in the downstream direction.

The computations show that the unsteady motion, as in the experiments $[8,9]$, is truly three dimensional. It is observed that the flow unsteadiness is sustainable even without any explicit random forcing and qualitatively similar unsteady structures are still generated. However, the discretization and round-off errors that, in fact, sup-

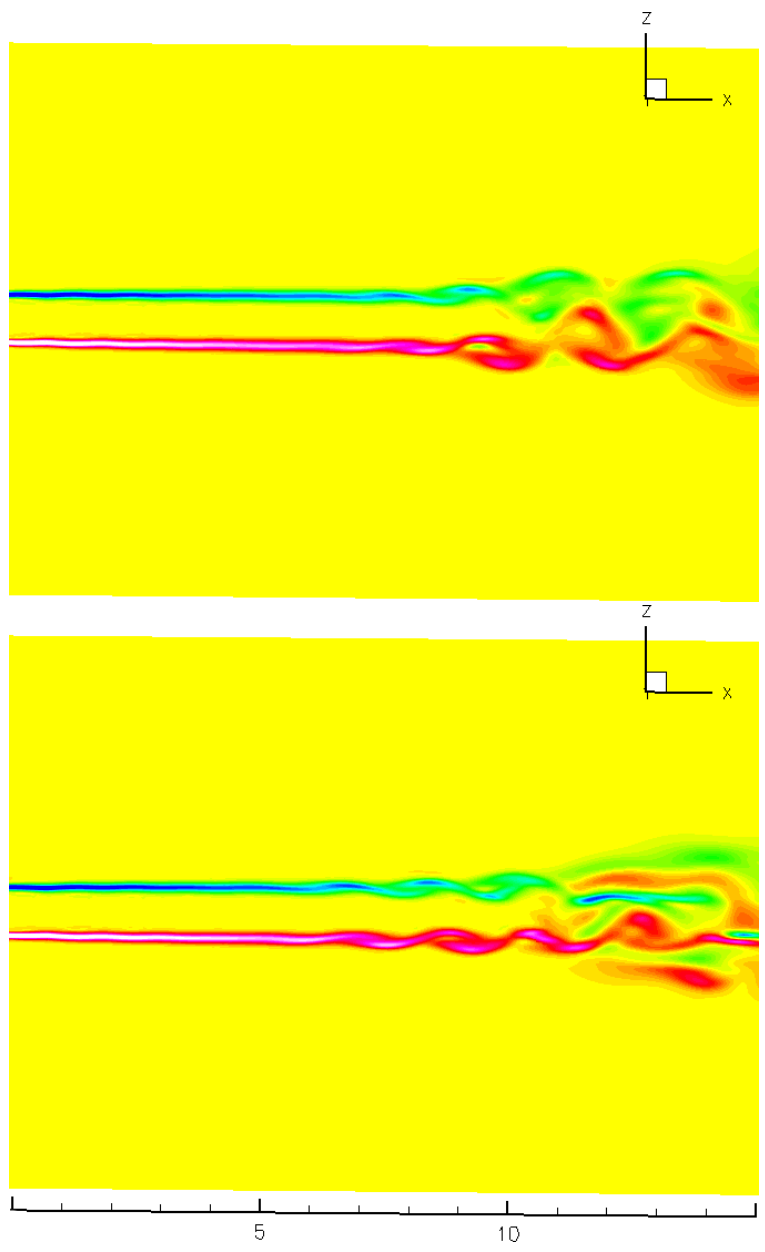

Figure 7: Instantaneous spanwise vorticity distribution in a spanwise plane; top, no forcing at $t=440$; bottom, random forcing at $t=890$

ply random forcing in this case are likely of too low an intensity to be truly representative of the experimental turbulence levels.

\subsection{Spectral Results}

Figure 10 shows the power spectral density (PSD) of the density fluctuations at three locations, $(x, y, z)$, in the flow field, namely $(2,0.48,0),(4,0.41,0)$, and $(12,0,0)$. The first two locations are in the jet shear layer, whereas the last one is on the centerline after the end of the potential core. The solid and long-dashed lines are simulation results with and without artificial forcing, respectively. The short-dashed line represents the experimental results $[8,9]$. The Strouhal number, consistent with the scaling used here, is defined as $S=f D / a_{\infty}$, where $\mathrm{f}$ is the dimensional frequency. It is quite common to use the jet velocity, rather than the ambient speed of sound, in the definition of the Strouhal numberdividing our Strouhal number by 1.2 (the present nondi- 


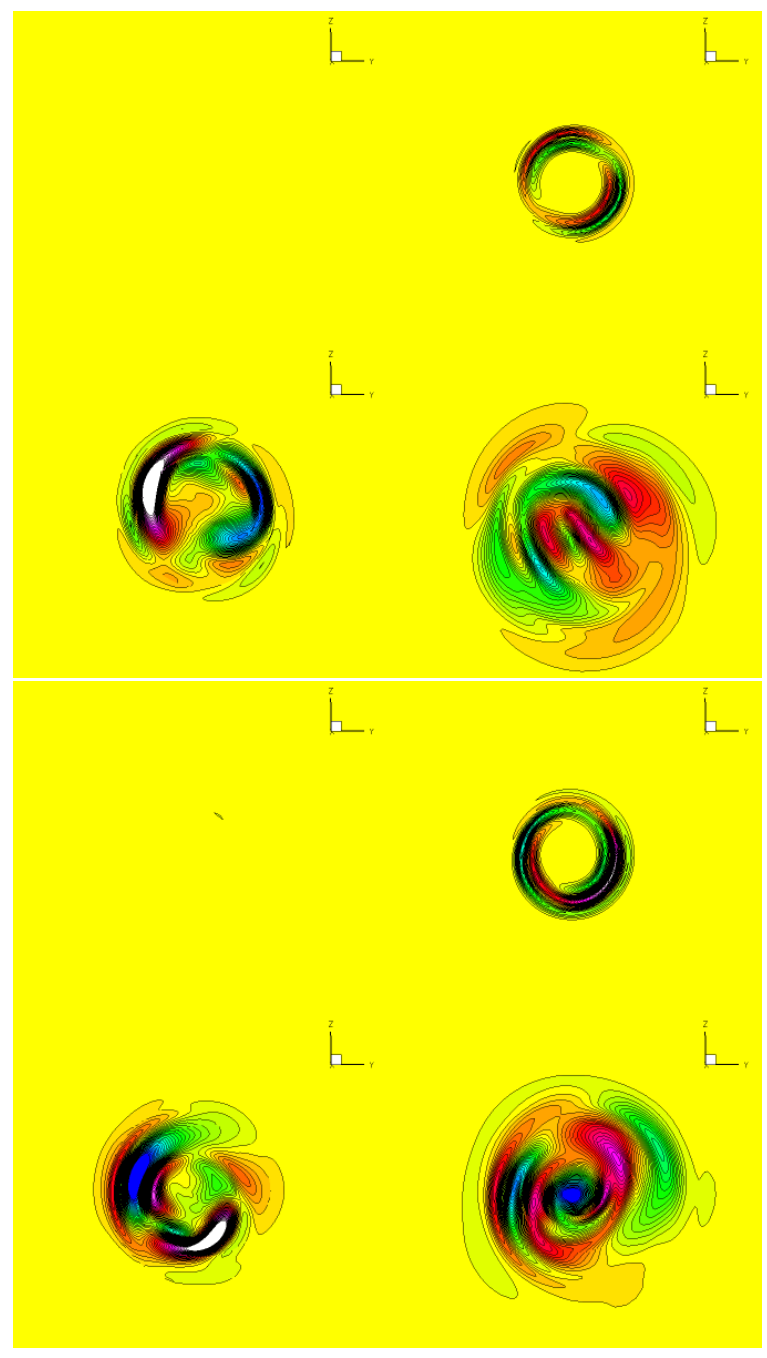

Figure 8: Instantaneous streamwise vorticity distribution in four streamwise planes; top, no forcing at $t=440$; bottom, random forcing at $t=890$

mensional jet velocity) converts it to the commonly used one.

The top plot in Fig. 10 is for the measuring point in the shear-layer closest to the nozzle, $(2,0.48,0)$. The broad hump in the experimental spectrum represents the hydrodynamic instability waves growing in the jet shear layer. It includes large-scale to small-scale disturbances. Due to limited grid resolution, and, of course, its general nature and underlying assumptions, the LES approach can not be expected capture the small-scale disturbances, nor maybe intermediate-scale ones. This is the reason for the sharp drop off in the numerical PSD curves. The agreement between the simulation and the experiments is, however, quite good for low Strouhal numbers (up to about 0.3 ), i.e., for large scale disturbances. The disagreement close to $S=0$ is related to the very short
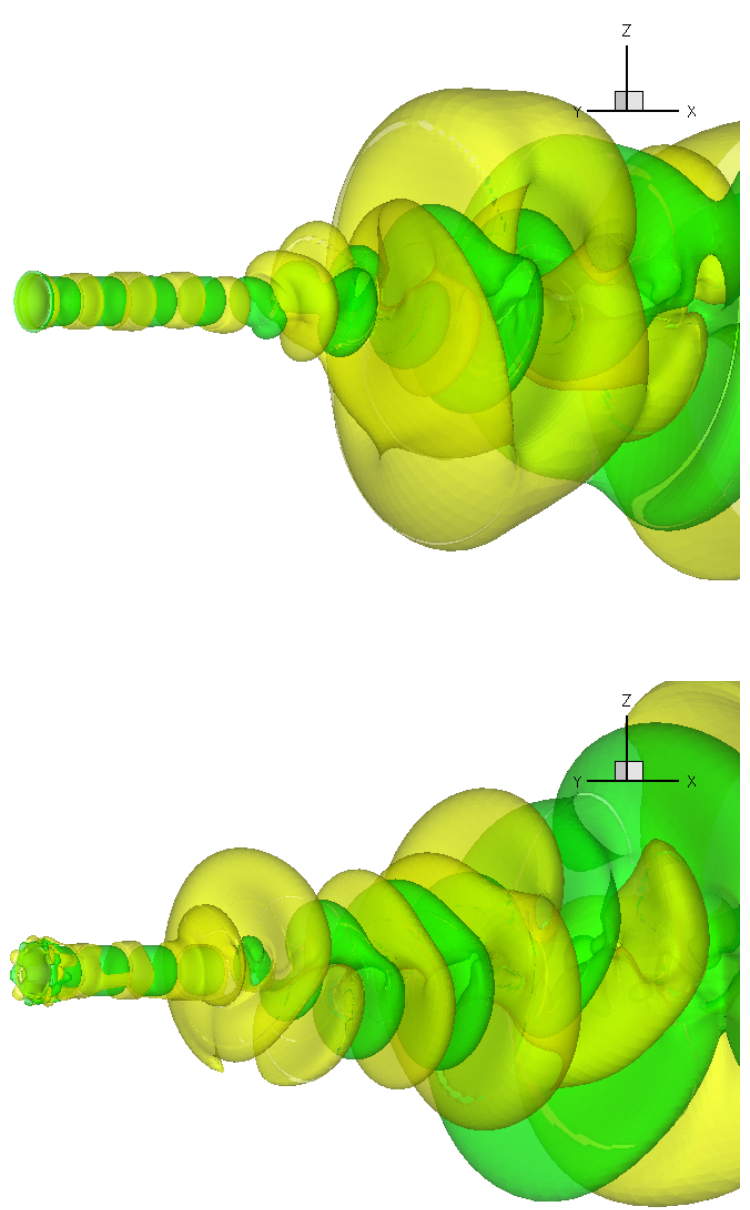

Figure 9: Instantaneous isobars; top, no forcing at $t=$ 440; bottom, random forcing at $t=890$

total observation time in the simulation compared to the experiment. The simulation results are quite insensitive to the artificial forcing. The middle plot in Fig. 10 corresponds to a location in the shear layer further downstream, $(4,0.41,0)$. The agreement between simulation and experiment is good also here for large-scale disturbances, and again the simulation results do not appear to be influenced much by the random forcing.

The bottom plot in Fig. 10 compares the simulated and experimental PSD for the density fluctuations at a point on the centerline even further downstream, (12, $0,0)$. At this streamwise location, there is no longer a potential core and the flow is highly unsteady and threedimensional. While the level of the simulation results are too high, the decay rate is comparable to the experimental data for $S$ less than about 0.6 , and then becomes steeper. This is an indication that for the current grid and 
LES approach, the cut-off Strouhal number is about 0.6. The much higher level of the numerical results, indicates that value of the Smargorinsky's eddy viscosity constant needs to be increased in this flow region, similar to our experience in the jet screech computations [30]. Again, there is practically no difference between the simulations with and without artificial random forcing.

\section{Concluding Remarks}

The present mixing-noise computation for a fully expanded supersonic circular jet demonstrates that the nearfield large-scale noise can be determined using a loworder (2nd order) finite-volume LES scheme, such as the current one. Generally, time-averaged results, such as for the streamwise velocity and radial density profiles agree well with existing experimental data. Near-field spectral results also agree well with the experiments in the expected range of lower Strouhal numbers. Instantaneous iso-surfaces of pressure show the radiating sound waves (eddy Mach wave radiation) originating from the largescale structures in the jet. Instantaneous streamwisevelocity and vorticity results demonstrate the highly unsteady and three-dimensional nature of the flow. The current results are in good qualitative agreement with what is generally understood about the flow physics of highspeed jets.

It is believed that the results can be further improved by lowering the damping in the numerical simulation by carefully reducing/optimizing the flux limiters and SGS parameters in the scheme. Further research in this area is currently being carried out.

\section{References}

[1] Tam, C. K. W., "Jet Noise Generated by Large Scale Coherent Motion," NASA RP-1258, pp. 311390 (1991).

[2] Seiner, J. M., "Advances in High Speed Jet Aeroacoustics," AIAA Paper 84-2275 (1984).

[3] Tam, C. K .W., “Supersonic Jet Noise,” Ann. Rev. Fluid Mech. vol. 27, pp. 17-43 (1995).

[4] Tam, C. K. W., "Computational Aeroacoustics: Issues and Methods," AIAA J. vol. 33, pp. 1788-1796 (1995).

[5] Tam, C. K. W., "Computational Aeroacoustics: An Overview of Computational Challenges and Applications," Inter. J. Comp. Fluid Dyn. vol. 18, pp. 547-567 (2004).

[6] Bailly, C. and Bogey, C., "Contributions of Computational Aeroacoustics to Jet Noise Research and Prediction," Inter. J. Comp. Fluid Dyn. vol. 18, pp. 481-491 (2004).
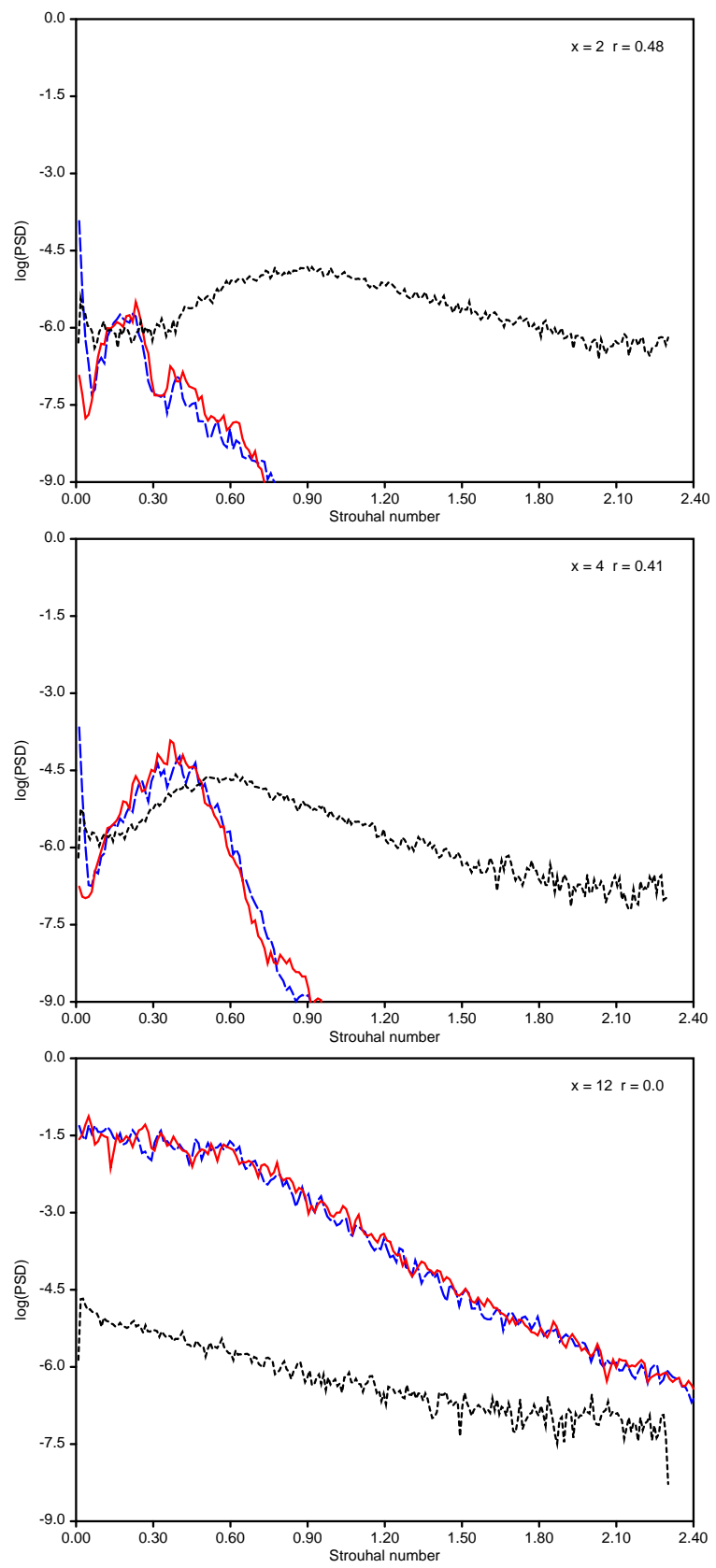

Figure 10: Density power spectral density (PSD) vs. Strouhal number at three locations; solid line: computation with artificial forcing, long-dashed line: computation with no forcing, short-dashed line: experiment 
[7] Panda, J. and Seasholtz, R. G., "Density Measurement in Underexpanded Supersonic Jets Using Rayleigh Scattering," AIAA Paper 98-0281 (1998)

[8] Panda, J. and Seasholtz, R. G., "Velocity and Temperature Measurement in Supersonic Free Jets Using Spectrally Resolved Rayleigh Scattering," AIAA Paper 99-0296 (1999).

[9] Panda, J. and Seasholtz, R. G., "Density Fluctuation Measurement in Supersonic Fully Expanded Jets Using Rayleigh Scattering," AIAA Paper 991870 (1999).

[10] Tam, C. K. W. and Chen, P., "Turbulent Mixing Noise from Supersonic Jets," AIAA J. vol. 32, pp. 1774-1780 (1994).

[11] Tam, C. K. W., Golebiowski, M. and Seiner, J. M., "On the Two Components of Turbulent Mixing Noise from Supersonic Jets," AIAA Paper 96-1716 (1996).

[12] Tam, C. K. W. and Pastouchenko, N. N., "On the Two Sources of Supersonic Jet Noise", AIAA Paper 2003-3163 (2003).

[13] Tam, C. K. W. and Auriault, L., "Mean Flow Refraction Effects on Sound Radiated from Localized Sources in a Jet," J. Fluid Mech. vol. 370, pp. 149174 (1998).

[14] Tam, C. K. W. and Auriault, L., "Jet Mixing Noise from Fine-Scale Turbulence," AIAA J. vol. 37, pp. 145-153 (1999).

[15] Tam, C. K. W. and Pastouchenko, N. N, "Noise from Fine-Scale Turbulence of Nonaxisymmetric Jets," AIAA J. vol. 40, pp. 456-464 (2003).

[16] Wundrow, D. W. and Khavaran, A., "On the Applicability of High-Frequency Approximations to Lilley's Equation," J. Sound Vibr. vol. 272, pp. 793830 (2004).

[17] Mankbadi, R. R., Hayer, M. E. and Povinelli, L. A., "Structure of Supersonic Jet Flow and Its Radiated Sound," AIAA J. vol. 32, pp. 897-906 (1994).

[18] Zhao, W., Frankel, S. H. and Mongeau, L.,"Large Eddy Simulation of Sound Radiation from a Subsonic Turbulent Jet." AIAA Paper 2000-2078 (2000).

[19] Bogey, C., Bailly, C. and Juve, D. "Noise Investigation of a High Subsonic, Moderate Reynolds Number Jet Using a Compressible Large Eddy Simulation," Theor. Comp. Fluid Dyn. vol. 16, pp. 273297 (2003).

[20] Uzun, A., Blaisdell, G. A. and Lyrintzis, A. S., "3D Large Eddy Simulation for Jet Aeroacoustics," AIAA Paper 2003-3322 (2003).
[21] Debonis, J. and Scott, J., "A Large Eddy Simulation of a Turbulent Compressible Round Jet," AIAA Paper 2001-2254 (2001).

[22] Loh, C. Y., Hultgren, L. S., and Jorgenson, P. C. E., "Near Field Screech Noise Computation for an Underexpanded jet by the CE/SE Method," AIAA Paper 2001-2252 (2001).

[23] Loh, C. Y. and Hultgren, L. S., "Jet Screech Noise Computation,” NASA/TM-2003-212626 (2003).

[24] Loh, C. Y. and Hultgren, L. S., "Computing Jet Screech - A Complex AeroacousticFeedback System", presented at the 2nd Int'l Conference on CFD (ICCFD2), also NASA/TM-2002-211807 (2002).

[25] Jorgenson, P. C. E. and Loh, C. Y., "Computing Axisymmetric Jet Screech Tones Using Unstructured Grids", AIAA Paper 2002-3889 (2002).

[26] Chang, S.-C., Wang, X.-Y. and Chow, C.-Y., "The Space-Time Conservation Element and Solution Element Method-A New High Resolution and Genuinely Multidimensional Paradigm for Solving Conservation Laws," J. Comp. Phys. vol. 159, pp. 89-136 (1999).

[27] Wang, X.-Y. and Chang S.-C., "A 2-D Non-splitting Unstructured Triangular Mesh Euler Solver Based on the Space-Time Conservation Element and Solution Element Method,' C.F.D. J. vol. 8, pp. 309-325 (1999).

[28] Karypis, G. and Kumar, V. "Multilevel k-way Partitioning Scheme for Irregular Graphs," Univ. of Minnesota Dept. of Comp. Sci./Army HPC Research Center Tech. Report 95-064 (1995).

[29] Anderson, D. A., Tannehill, J. C. and Pletcher, R. H., Computational Fluid Mechanics and Heat Transfer, MacGraw-Hill Book Company (1984).

[30] Loh, C. Y., Himansu, A. and Hultgren, L. S., "A 3D Navier-Stokes Solver with Unstructured Hexahedral Grid for Computation of Nearfield Jet Screech Noise," AIAA Paper 2003-3207 (2003).

[31] Lesieur, M. and Métais, O., "New Trends in LargeEddy Simulation of Turbulence," Ann. Rev. Fluid Mech. vol. 28, pp. 45-82 (1996).

[32] Fureby, C. and Grinstein, F. F., "Monotonically Integrated Large Eddy Simulation of Free Shear Flows," AIAA J., vol. 37, pp. 544-556 (1999).

[33] Loh, C. Y., “On a Non-Relecting Boundary Condition for Hyperbolic Conservation Laws," AIAA Paper 2003-3975 (2003).

[34] Loh, C. Y. and Jorgenson, P. C. E., "A Robust Absorbing Boundary Condition for Compressible Flows," AIAA Paper 2005-4716 (2005). 


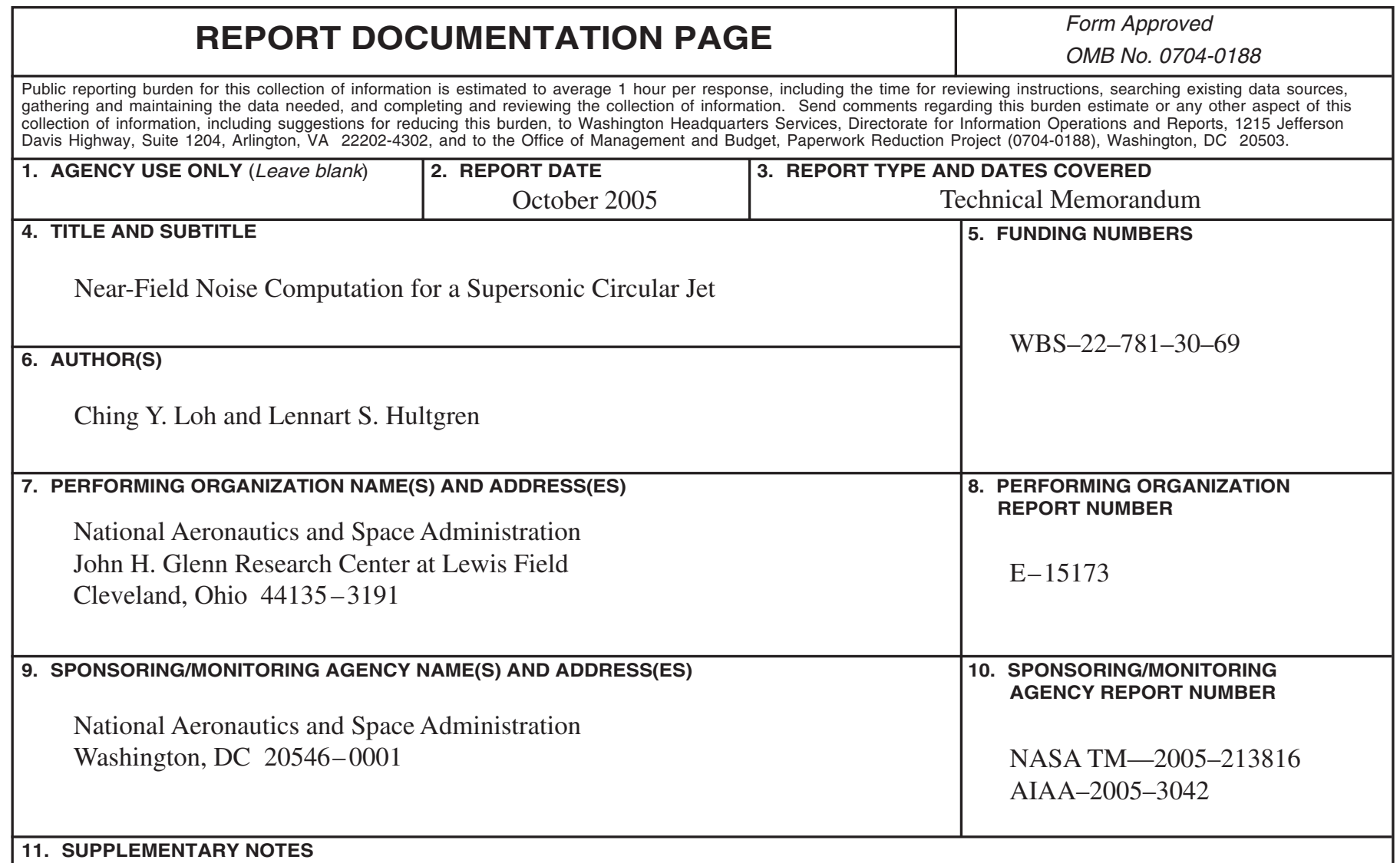

Prepared for the 11th Aeroacoustics Conference cosponsored by the American Institute of Aeronautics and Astronautics and the Confederation of European Aerospace Societies, Monterey, California, May 23-25, 2005. Ching Y. Loh, Taitech, Inc., 1430 Oak Court, Suite 301, Beavercreek, Ohio 45430; and Lennart S. Hultgren, NASA Glenn Research Center. Responsible person, Ching Y. Loh, organization code RTS, 216-433-3981.

\begin{tabular}{l} 
12a. DISTRIBUTION/AVAILABILITY STATEMENT \\
Unclassified - Unlimited \\
Subject Categories: 02,71 , and 64 \\
Available electronically at http://gltrs.grc.nasa.gov \\
This publication is available from the NASA Center for AeroSpace Information, 301-621-0390. \\
\hline 13. ABSTRACT (Maximum 200 words)
\end{tabular}

A fully expanded, high-Reynolds-number, supersonic circular jet of Mach number 1.4 is simulated, using a 3-D finite-volume Navier-Stokes solver, with emphasis on the near field noise. The numerical results are generally in good agreement with existing experimental findings.

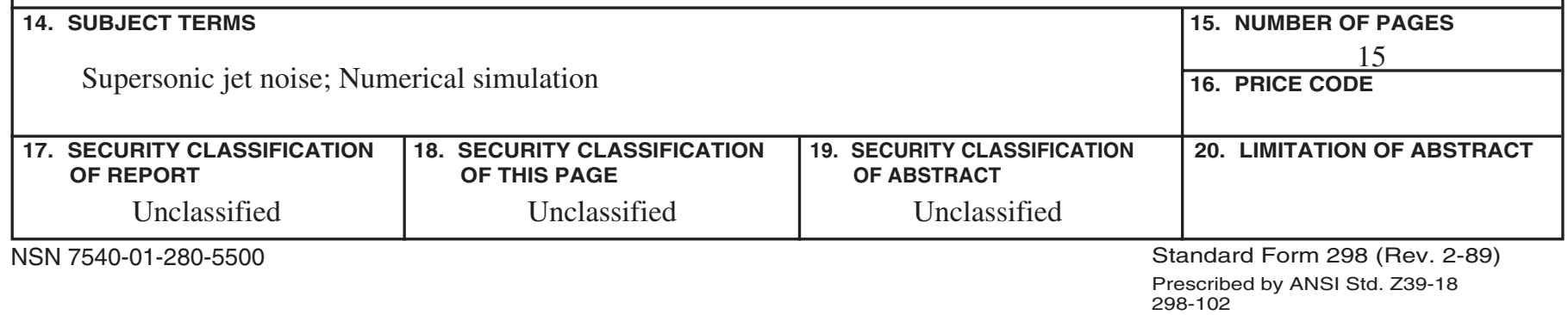



